14

\title{
Плотность незаполненных электронных состояний осажденных в вакууме пленок диоктил-замещенного и дифенил-замещенного перилен-дикарбоксимида
}

\author{
(C) А.С. Комолов ${ }^{1}$, Э.Ф. Лазнева ${ }^{1}$, Н.Б. Герасимова ${ }^{1}$, Ю.А. Панина ${ }^{1}$, \\ А.В. Барамыгин ${ }^{1}$, Г.Д. Зашихин ${ }^{1}$, C.А. Пшеничнюк ${ }^{1,2}$ \\ ${ }^{1}$ Санкт-Петербургский государственный университет, \\ Санкт-Петербург, Россия \\ ${ }^{2}$ Институт физики молекул и кристаллов УНЦ РАН, \\ Уфра, Россия \\ E-mail: a.komolov@spbu.ru
}

(Поступила в Редакцию 14 апреля 2016 г.)

\begin{abstract}
Приведены результаты исследования плотности незаполненных электронных состояний в энергетическом диапазоне от 5 до $20 \mathrm{eV}$ выше энергии Ферми $\left(E_{\mathrm{F}}\right)$ в сверхтонких пленках диоктил-замещенного и дифенилзамещенного перилен-дикарбоксимида. Экспериментальные результаты получены путем регистрации тока вторичных низкоэнергетических электронов с использованием методики спектроскопии полного тока. Теоретический анализ включал в себя расчет энергий и пространственного распределения орбиталей исследованных молекул методом теории функционала плотности и последующее масштабирование рассчитанных значений энергий орбиталей согласно процедуре, хорошо зарекомендовавшей себя ранее при исследованиях малых сопряженных органических молекул. Обнаружено, что для обоих видов исследованных пленок при энергиях ниже $8 \mathrm{eV}$ над $E_{\mathrm{F}}$ расположены по два основных максимума плотности незаполненных электронных состояний, образованных преимущественно $\pi^{*}$-орбиталями молекул. Более высоколежащие максимумы имеют преимущественно $\sigma^{*}$ характер. Проведен анализ влияния диоктил- и дифенил-замещающих групп на плотность незаполненных электронных состояний при сравнении результатов для исследованных видов пленок. В случае $\pi^{*}$-максимумов наблюдается относительный сдвиг, около $1 \mathrm{eV}$, по энергии. В области $\sigma^{*}$-электронных состояний наблюдается незначительная перестройка структуры максимумов.
\end{abstract}

Работа выполнена при поддержке научного гранта СПбГУ 11.38.219.2014, РФФИ (14-03-00087 и 15-29-05786).

DOI: $10.21883 /$ FTT.2017.02.44068.142

\section{1. Введение}

Электронные свойства пограничных структур на основе сопряженных органических материалов представляют значительный интерес в связи с возможностями их использования в устройствах органической электроники [1-3]. Возможным вариантом оптимизации приборных характеристик органических электронных устройств является введение в состав молекул замещающих групп таких, как алкильные цепочки различной длины либо поляризующие заместители [4,5]. Это представляет и значительный фундаментальный интерес в плане установления закономерностей формирования энергетических зон электронных состояний в органических полупроводниках. Достаточно перспективными считают материалы на основе молекул замещенных периленов, такие как диоктил-замещенный перилен-дикарбоксимид $($ PTCDI-C 8 ) (рис. 1, сверху) [6]. В результате исследований методами электронной спектроскопии были определены максимумы вакантных энергетических состояний в зоне проводимости, соответствующие ароматическому периленовому остову молекулы и фрагментам заместителей - карбоновых кислот [6-8].
Одним из экспериментальных подходов к изучению плотности незаполненных электронных состояний (DOUS) является методика спектроскопии полного тока (CПТ) $[9,10]$, в которой используется тестирующий пучок низкоэнергетических электронов и производится регистрация интегрального тока вторичных электронов. Одновременный теоретический анализ с использованием результатов $a b$ initio расчетов позволяет определить природу обнаруженных максимумов DOUS. Ранее мы проводили исследование модификации тонкой структуры спектров полного тока (ТССПТ) пленок PTCDI-C 8 и дифенил-замещенного перилендикарбоксимида (PTCDI-Ph) в процессе их осаждения на поверхности окисленных кремния и германия [11-13]. Было показано, что при толщине покрытия более $6 \mathrm{~nm}$ ТССПТ практически не зависит от материала подложки и является характеристикой исследованного органического материала. В данной статье приведены результаты исследования плотности незаполненных электронных состояний (DOUS) пленок PTCDI-C 8 и PTCDI-Ph (рис. 1, снизу) в диапазоне от 5 до $20 \mathrm{eV}$ выше $E_{\mathrm{F}}$, полученной на основе экспериментальных ТССПТ и на основе результатов теоретических расчетов с использованием методов теории функционала плотности (DFT). 


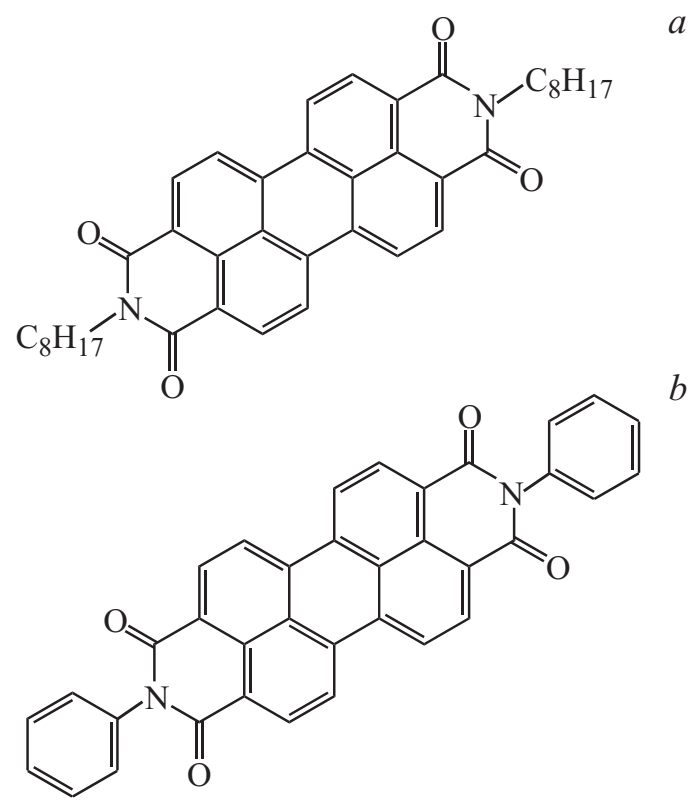

$a$

Рис. 1. Структурная формула молекул $N, N^{\prime}$-диоктил-3,4,9,10перилен-дикарбоксимид (PTCDI-C 8 ) (a) и $N, N^{\prime}$-дифенил3,4,9,10-перилен-дикарбоксимид (PTCDI-Ph) (b).

\section{2. Эксперимент и метод расчета}

Для приготовления пленочных образцов использовали реактивы $N, N^{\prime}$-диоктил-3,4,9,10-перилен-дикарбоксимид $\left(\mathrm{PTCDI}-\mathrm{C}_{8}\right)$ и $N, N^{\prime}$-дифенил-3,4,9,10-перилен-дикарбоксимид (PTCDI-Ph) (рис. 1), приобретенные у производителя Sigma-Aldrich. После предварительного обезгаживания реактивов in situ в вакуумной камере при базовом давлении $10^{-6} \mathrm{~Pa}$ при температуре $100^{\circ} \mathrm{C}$ проводили термическое осаждение органического материала, PTCDI-C 8 или PTCDI-Ph, в вакууме на поверхность подложки со скоростью примерно $0.1 \mathrm{~nm} / \mathrm{min}$, как это описано более подробно в работе [13]. Такая процедура осаждения, без дополнительного нагрева подложки или отжига осажденного органического слоя, обычно приводит к формированию неупорядоченных пленок замещенных периленов [14]. В качестве подложки использовали пластины германия с реальным окислом $\left(\mathrm{GeO}_{2}\right) \mathrm{Ge}$, очищенные in situ импульсными прогревами до $400^{\circ} \mathrm{C}$ с использованием сфокусированного излучения $300 \mathrm{~W}$ металлогалогенной лампы высокого давления. Согласно результатам контроля методом рентгеновской электронной спектроскопии, толщина слоя оксида германия составляла менее $5 \mathrm{~nm}$, как это обсуждалось более подробно в работе [13].

Толщину органических пленок PTCDI-C 8 и PTCDI-Ph в процессе осаждения доводили до $6-8 \mathrm{~nm}$. Контроль толщины и измерения электронной структуры зоны проводимости исследованных пленок производили методом СПТ, согласно которому параллельный пучок электронов площадью $0.2-0.4 \mathrm{~mm}^{2}$ направляется по нормали к исследуемой поверхности, и регистрируется производ- ная по энергии от полного тока $S(E)$, проходящего через образец, как функция энергии падающих электронов, которую варьируют в пределах от 0 до $25 \mathrm{eV}[9,10]$. Инструментально СПТ реализована с использованием стандартного анализатора дифракции медленных электронов. При этом в случае неупорядоченных поверхностей картина дифракционных рефлексов не наблюдается, а интегральный ток вторичных электронов может быть измерен. Зависимость $S(E)$, тонкая структура спектров полного тока (ТССПТ), отражает изменения значения коэффициента упругого рассеяния падающих низкоэнергетических электронов от поверхности исследуемого образца, которые связаны с зависимостью плотности незаполненных электронных состояний (DOUS) от энергии, как было показано ранее для широкого круга неорганических и органических полупроводниковых материалов $[9,15-17]$.

Теоретический анализ DOUS исследованных пленок проводили с использованием квантово-химических расчетов энергий орбиталей исследованных методами теории функционала плотности (DFT) с помощью гибридного функционала B3LYP [18] в базисе 6-31G(d), реализованными в программном пакете Gaussian [19]. На основе изображений пространственного распределения орбиталей определяли их $\pi^{*}$ - и $\sigma^{*}$-характер, так как $\sigma^{*}$-орбиталь симметрична относительно линии связи, а $\pi^{*}$-орбиталь симметрична относительно плоскости, проходящей через линию связи. Для уточнения рассчитанных значений энергий орбиталей (virtual orbital energies, VOE) использовали подход, предложенный в работе [20], состоящий в корректировке масштабировании согласно результатам исследований DOUS для малых сопряженных органических молекул [20-23]. Для дальнейшего анализа использовали отмасштабированные значения энергий орбиталей (scaled virtual orbital energies, SVOE). Для масштабирования $\pi^{*}$-молекулярных орбиталей использовали выражение $\mathrm{SVOE}=(\mathrm{VOE}+1.14) / 1.24$, а для $\sigma^{*}$-орбиталей - выражение SVOE $=(\mathrm{VOE}+0.97) / 0.79[20,22,24]$.

\section{3. Результаты и обсуждение}

Для построения экспериментальной DOUS использовали зависимости ТССПТ для пленок PTCDI-C 8 и PTCDI-Ph толщиной $6 \mathrm{~nm}$ [13]. При такой толщине исследованных органических пленок ТССПТ практически не зависит от материала подложки и является характеристикой исследованного органического материала [11-13]. Действительно, при физической адсорбции органических молекул на подложку влияние последней в значительном количестве случаев распространяется на пограничную область в органической пленке толщиной не более $2 \mathrm{~nm}[6,9,25]$. При этом в некоторых случаях, как например в случае пленок замещенных периленов на поверхности оксида цинка, первый молекулярный слой пленки может даже состоять из молекулярных ионов, 


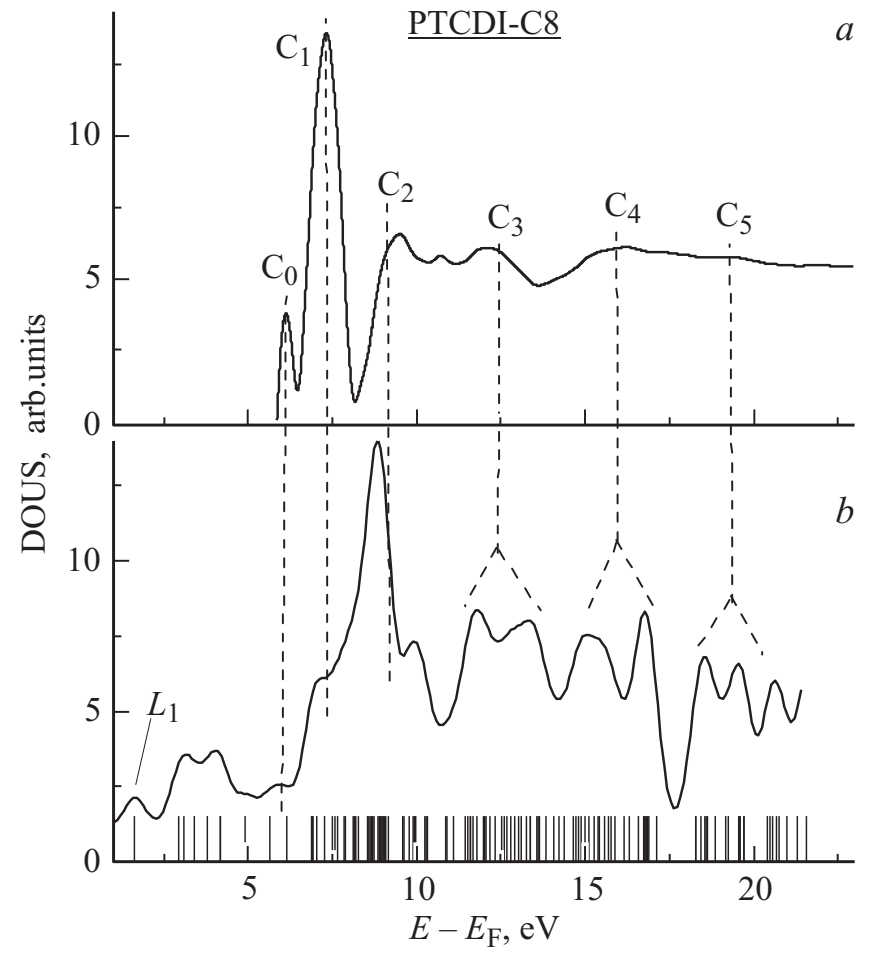

Pис. 2. $a-$ DOUS пленок PTCDI-C $\mathrm{C}_{8}$, построенная на основе экспериментальных ТССПТ. $\mathrm{C}_{0}-\mathrm{C}_{5}-$ обозначения основных максимумов. $b-$ результаты расчетов энергий орбиталей молекул PTCDI-C 8 с использованием методов DFT на уровне B3LYP/6-31G(d) (вертикальные сплошные линии) и DOUS, построенная на основе результатов расчетов. $L_{1}-$ самый низколежащий максимум в зоне проводимости (LUMO) пленок PTCDI-C 8 . Вертикальные штриховые линии показаны для удобства сравнения положений максимумов.

однако при увеличении толщины органического покрытия влияние подложки значительно уменьшается [26]. Как было показано в ряде работ, структура максимумов DOUS соответствует структуре максимумов ТCCПТ и может быть построена в виде производной $S(E)$ с обратным знаком: $-d S(E) / d E[9,15]$.

DOUS пленок PTCDI-C 8 , построенная на основе экспериментальных результатов, представлена на pис. 2, a. Для нее характерны максимумы $\mathrm{C}_{0}-\mathrm{C}_{5}$, pacположенные в диапазоне энергий от 5 до $20 \mathrm{eV}$ выше уровня Ферми. Проведен расчет энергий орбиталей молекул PTCDI-C 8 методом теории функционала плотности (DFT) на уровне B3LYP/6-31G(d) с последующим масштабированием [20-22], как обсуждалось в разделе 2. Путем построения свертки рассчитанных энергий орбиталей с функцией Гаусса, полушириной $0.5 \mathrm{eV}$, построена зависимость DOUS. Следует заметить, что расчет энергий орбиталей производился для молекул в газовой фазе. Поэтому для сравнения построенной так теоретической DOUS зависимости с экспериментальной, измеренной для твердотельной фазы - пленок - следует учесть дополнительно и величину энергии поляризации среды.
Для области энергий вакантных орбиталей это обычно делают путем сдвига рассчитанной DOUS на $1-1.5 \mathrm{eV}$ в сторону меньших значений энергий электрона [22,27]. При сравнении теоретической и экспериментальной DOUS зависимостей для пленок наблюдается достаточно хорошее соответствие энергетического расположения в исследованном диапазоне от 5 до $20 \mathrm{eV}$ выше уровня Ферми (рис. 2). Максимумам $\mathrm{C}_{0}$ и $\mathrm{C}_{1}$ экспериментальной DOUS соответствуют отдельные максимумы на теоретической DOUS, максимуму $\mathrm{C}_{2}$ соответствует наиболее интенсивный максимум теоретической DOUS, a более широким экспериментальным максимумам $\mathrm{C}_{3}-\mathrm{C}_{5}$ соответствуют парные вершины на теоретической зависимости (рис. 2).

Рассчитанное пространственное распределение орбиталей можно было визуализировать с помощью используемого программного пакета, что позволило установить их $\pi^{*}$ - или $\sigma^{*}$-характер. Для пленок PTCDI-C 8 установлено, что при энергиях менее $7.5 \mathrm{eV}$ над уровнем $E_{\mathrm{F}}$ расположены преимущественно $\pi^{*}$-орбитали, а в диапазоне энергий от 7.5 до $9.5 \mathrm{eV}$ располагается переходная область, в которой присутствуют как $\pi^{*}$-, так и $\sigma^{*}$-орбитали. Таким образом, максимумы DOUS $\mathrm{C}_{0}$, и низкоэнергетический компонент максимума $\mathrm{C}_{1}$

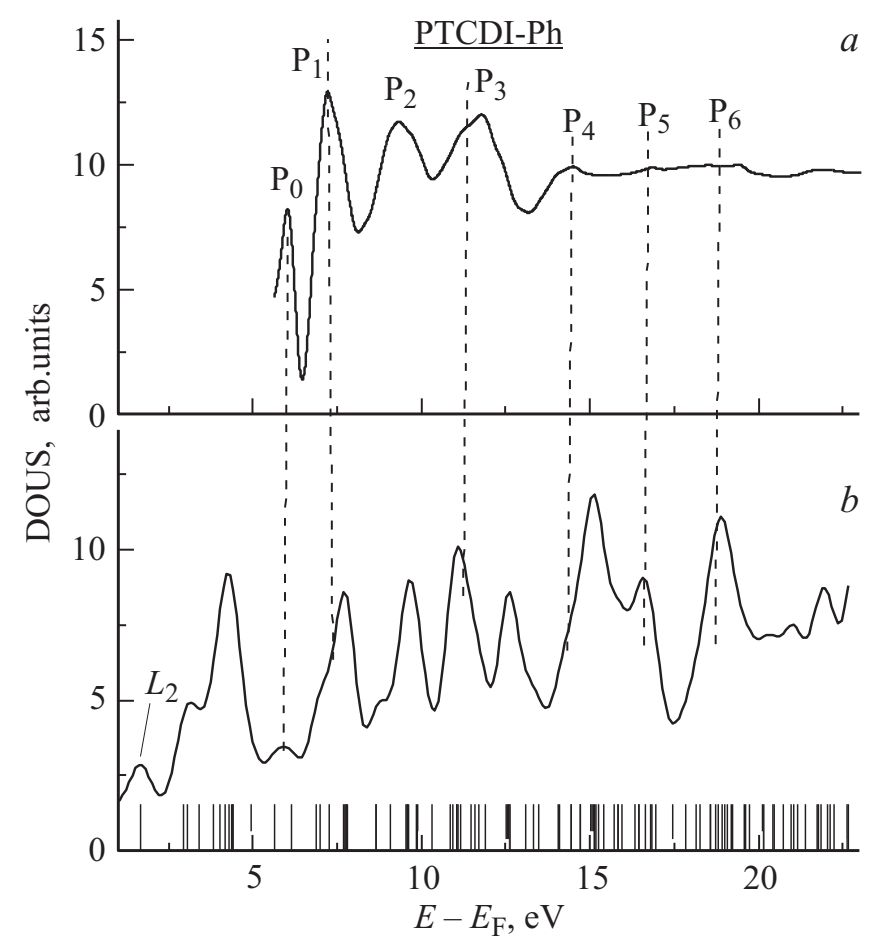

Рис. 3. $a-$ DOUS пленок PTCDI-Ph, построенная на основе экспериментальных ТССПТ. $\mathrm{P}_{0}-\mathrm{P}_{6}-$ обозначения основных максимумов. $b-$ результаты расчетов энергий орбиталей молекул PTCDI-Ph с использованием методов DFT на уровне B3LYP/6-31G(d) (вертикальные сплошные линии) и DOUS, построенная на основе результатов расчетов. $L_{2}-$ самый низколежащий максимум в зоне проводимости (LUMO) пленок PTCDI-Ph. Вертикальные пунктирные линии - см. подпись к рис. 2. 
(рис. 2) сформированы $\pi^{*}$-орбиталями, высокоэнергетический компонент максимума $\mathrm{C}_{1}$ и максимум $\mathrm{C}_{2}$ имеют смешанную, $\pi^{*}$ и $\sigma^{*}$, природу. Более высоколежащие максимумы $\mathrm{C}_{3}-\mathrm{C}_{5}$ (рис. 2) имеют преимущественно $\sigma^{*}$-характер, однако при энергиях около 16 и $20.5 \mathrm{eV}$ расположены и несколько орбиталей $\pi^{*}$-типа. О наличии максимумов DOUS, имеющих смешанную, $\pi^{*}$ и $\sigma^{*}$-, природу упоминалось в литературе ранее [28,29]. В том числе при фторозамещении молекул бензола было обнаружено, что происходила стабилизация части низколежащих $\sigma^{*}$-орбиталей. При этом энергетическое положение $\sigma^{*}$-максимума, образовавшегося в результате стабилизации, оказывалось даже ниже, чем у максимума $\pi^{*}$ [28]. Смешанные, $\pi^{*}$ - и $\sigma^{*}$-, энергетические области при энергиях около $10 \mathrm{eV}$ выше $E_{\mathrm{F}}$ шириной в несколько $\mathrm{eV}$ были обнаружены нами в зависимостях DOUS пленок фталоцианинов меди и замещенных фуллеренов [24,30].

DOUS пленок PTCDI-Ph, построенная на основе экспериментальных результатов, имеет максимумы $\mathrm{P}_{0}-\mathrm{P}_{6}$ в диапазоне энергий от 5 до $20 \mathrm{eV}$ выше уровня Ферми (рис. 3,a). Расчет энергий орбиталей и теоретической DOUS для этих пленок (рис. $3, b$ ) произведен методом DFT с учетом процедуры масштабирования и энергии поляризации среды [22,27], аналогично рассмотренному в этом разделе случаю пленок PTCDI-C 8 . Обнаружено достаточно хорошее соответствие основных максимумов $\mathrm{P}_{0}-\mathrm{P}_{6}$ экспериментальной DOUS и теоретически рассчитанной DOUS (рис. 3). Действительно, каждому максимуму $\mathrm{P}_{0}-\mathrm{P}_{6}$ соответствует максимум в рассчитанной DOUS практически во всем рассмотренном диапазоне энергий от 5 до $20 \mathrm{eV}$ выше $E_{\mathrm{F}}$. Некоторые отклонения от этого наблюдаются в области энергетического положения двух достаточно широких максимумов $\mathrm{P}_{3}$ и $\mathrm{P}_{4}$, где теоретическая DOUS представлена тремя максимумами. Рассчитанное пространственное распределение орбиталей позволило установить их $\pi^{*}$ - или $\sigma^{*}$-характер. Так для пленок PTCDI-Ph при энергиях менее $8 \mathrm{eV}$ над уровнем $E_{\mathrm{F}}$ расположены преимущественно $\pi^{*}$-орбитали. В этой энергетической области расположены максимумы экспериментальной DOUS $\mathrm{P}_{0}$ и $\mathrm{P}_{1}$. Области смешивания $\pi^{*}$ - и $\sigma^{*}$-орбиталей соответствует максимум $\mathrm{P}_{2}$, а более высоколежащие максимумы $\mathrm{P}_{3}-\mathrm{P}_{6}$ (рис. 3) имеют преимущественно $\sigma^{*}$-характер.

Сравним DOUS двух исследованных видов пленок $\mathrm{PTCDI}_{-}$и PTCDI-Ph. В области энергий менее $9 \mathrm{eV} \pi^{*}$-максимумы пленки РTCDI-Ph расположены на 0.5-1 eV ниже, чем в случае пленки PTCDI-C 8 (рис. 2 и 3). В области энергий от 8 до $13 \mathrm{eV}$, соответствующей низколежащим $\sigma^{*}$-максимумам $\mathrm{C}_{2}, \mathrm{C}_{3}$ и $\mathrm{P}_{2}, \mathrm{P}_{3}$, различия в DOUS двух видов пленок выражены слабо. А в области от 13 до $17 \mathrm{eV}$ наблюдаются различия в DOUS, на которые указывает сдвиг максимума относительно соответствующего ему максимума $\mathrm{C}_{4}$ в сторону меньших значений энергии электрона. Влияние заместителей на DOUS пленок малых сопряженных органических молекул в виде относительного сдвига $\pi^{*}$-максимумов и незначительной перестройки $\sigma^{*}$-максимумов наблюдалось нами ранее при исследованиях пленок фталоцианинов меди и олигомеров фенилен-винилена $[10,30]$. Согласно литературным данным $[28,31]$ стабилизацию $\pi^{*}$-максимумов можно связать с переносом электронной плотности из сопряженного остова органической молекулы в направлении периферийных замещающих групп.

\section{4. Выводы}

На основе результатов СПТ экспериментов и теоретических расчетов на уровне B3LYP/6-31G(d) с дополнительной корректировкой установлены основные максимумы DOUS пленок PTCDI-C 8 и PTCDI-Ph в диапазоне от 5 до $20 \mathrm{eV}$ выше $E_{\mathrm{F}}$. При энергиях ниже $8 \mathrm{eV}$ над $E_{\mathrm{F}}$ в пленках расположены по два максимума DOUS, образованных преимущественно $\pi^{*}$-орбиталями молекул. Максимумы DOUS, расположенные при энергиях от 9.5 до $20 \mathrm{eV}$, имеют $\sigma^{*}$-природу. $\pi^{*}$-максимумы пленки PTCDI-Ph расположены на $0.5-1 \mathrm{eV}$ ниже соответствующих максимумов пленки PTCDI-C 8 . В области энергий от 8 до $13 \mathrm{eV}$, соответствующей низколежащим $\sigma^{*}$-максимумам, различия в DOUS двух видов пленок выражены слабо, а в области от 13 до $17 \mathrm{eV}$ различия обусловлены главным образом сдвигом основного максимума DOUS.

\section{Список литературы}

[1] J. Jo, J.-R. Pouliot, D. Wynands, S.D. Collins, J.Y. Kim, T.L. Nguyen, H.Y. Woo, Y. Sun, M. Leclerc, A.J. Heeger. Adv. Mater. 25, 34, 4783 (2013).

[2] Y.N. Luponosov, N.M. Surin, D.K. Susarova, M.I. Buzin, D.V. Anokhin, D.A. Ivanov, P.A. Troshin, S.A. Ponomarenko. Organic Photonics Photovoltaics 3, 1, 148 (2015).

[3] I.E. Gracheva, V.A. Moshnikov, E.V. Maraeva, S.S. Karpova, O.A. Alexsandrova, N.I. Alekseyev, V.V. Kuznetsov, G. Olchowik, K.N. Semenov, A.V. Startseva, A.V. Sitnikov, J.M. Olchowik. J. Non-Cryst. Solids 358, 2, 433 (2012).

[4] M. Marks, S. Sachs, C.H. Schwalb, A. Schöll, U. Höfer. J. Chem. Phys. 139, 12, 124701 (2013).

[5] P.A. Troshin, D.K. Susarova, E.A. Khakina, A.A. Goryachev, O.V. Borshchev, S.A. Ponomarenko, V.F. Razumov, N. Serdar Sariciftci. J. Mater. Chem. 22, 18433 (2012).

[6] I.G. Hill, J. Schwartz, A. Kahn. Organic Electr. 1, 5 (2000).

[7] N. Hiroshiba, R. Hayakawa, T. Chikyow. Phys. Chem. Chem. Phys. 13, 6280 (2011).

[8] J. Taborski, P. Vaterlein, U. Zimmermann, E. Umbach. J. Electr. Spec. Rel. Phen. 75, 129 (1995).

[9] A.S. Komolov, E.F. Lazneva, S.N. Akhremtchik. App. Surf. Sci. 256, 2419 (2010).

[10] A.S. Komolov, E.F. Lazneva, S.N. Akhremtchik, N.S. Chepilko, A.A. Gavrikov. J. Phys. Chem. C 117, 24, 12633 (2013).

[11] А.С. Комолов, Э.Ф. Лазнева, Н.Б. Герасимова, Ю.А. Панина, А.В. Барамыгин, А.Д. Овсянников. ФТТ 57, 1445 (2015). 
[12] А.С. Комолов, Э.Ф. Лазнева, Н.Б. Герасимова, Ю.А. Панина, А.В. Барамыгин, Г.Д. Зашихин, С.А. Пшеничнюк. ФТТ 58, 367 (2016).

[13] А.С. Комолов, Э.Ф. Лазнева, Н.Б. Герасимова, Ю.А. Панина, А.В. Барамыгин, С.А. Пшеничнюк. ФТТ 58, 9, 1836 (2016).

[14] S. Heutz, A.J. Ferguson, G. Rumbles, T.S. Jones. Organic Electr. 3, 119 (2002).

[15] I. Bartos. Progr. Surf. Sci. 59, 197 (1998).

[16] A.S. Komolov, E.F. Lazneva, Y.G. Aliaev, S.A. Akhremchik, F.S. Kamounah, J. Mortenson, K. Schaumburg. J. Molec. Struc. 744/747, 145 (2005).

[17] S.A. Pshenichnyuk, A.S. Komolov. J. Phys. Chem. A 116, 1, 761 (2012).

[18] A.D. Becke. J. Chem. Phys. 98, 5648 (1993).

[19] M.J. Frisch, G.W. Trucks, H.B. Schlegel, G.E. Scuseria, M.A. Robb, J.R. Cheeseman, G. Scalmani, V. Barone, B. Mennucci, G.A. Petersson et al. Gaussian 09, Revision D.01, Gaussian, Inc., Wallingford CT (2009).

[20] P.D. Burrow, A. Modelli. SAR and QSAR in Env. Res. 24, 647 (2013).

[21] С.А. Пшеничнюк, А.В. Кухто, И.Н. Кухто, А.С. Комолов. ЖТФ 81, 6, 8 (2011).

[22] A.M. Scheer, P.D. Burrow. J. Phys. Chem. B 110, 17751 (2006).

[23] N.L. Asfandiarov, S.A. Pshenichnyuk, A.S. Vorob'ev, E.P. Nafikova, A. Modelli. Rapid Commun. Mass Spectrom. 29, 910 (2015).

[24] A.S. Komolov, E.F. Lazneva, N.B. Gerasimova, Yu.A. Panina, G.D. Zashikhin, A.V. Baramygin, P. Si, S.N. Akhremtchik, A.A. Gavrikov. J. Electron Spectr. Rel. Phen. 205, 52 (2015).

[25] L. Grzadziel, M. Krzywiecki, H. Peisert, T. Chassé, J. Szuber. Organic Electron. 13, 10, 1873 (2012).

[26] M. Gruenewald, L.K. Schirra, P. Winget, M. Kozlik, P.F. Ndione, A.K. Sigdel, J.J. Berry, R. Forker, J.-L. Brédas, T. Fritz, O.L.A. Monti. J. Phys. Chem. C 119, 4865 (2015).

[27] I.G. Hill, A. Kahn, J. Cornil, D.A. dos Santos, J.L. Bredas. Chem. Phys. Lett. 317, 444 (2000).

[28] A.P. Hitchcock, P. Fischer, A. Gedanken, M.B. Robin. J. Phys. Chem. 91, 531 (1987).

[29] J.G. Chen. Surf. Sci Rep. 30, 1 (1997).

[30] А.С. Комолов, Э.Ф. Лазнева, Н.Б. Герасимов, Ю.А. Панина, А.В. Баратыгин, Я.В. Ахремчик, А.В. Поволоцкий. ФТТ 56, 2464 (2014).

[31] J. Cornil, D.A. Dos Santos, D. Beljonne, J.L. Bredas. J. Phys. Chem. 99, 5604 (1995). 